\title{
EVALUACIÓN DEL CONTROL INTERNO EN EL PROCESO DE TESORERÍA DE LAS PEQUEÑAS Y MEDIANAS EMPRESAS DE MEDELLÍN
}

\author{
Evaluation of internal control in the treasury process at small and medium-sized companies \\ in Medellin
}

\author{
Daniel Alberto Grajales-Gaviria \\ MSc en Educación, Instituto Tecnológico Metropolitano ITM, Medellín-Colombia, \\ grajalesdaniel@gmail.com \\ Orlando Carmelo Castellanos-Polo \\ MSc en Auditoria Internacional, Corporación Universitaria Americana, Medellín-Colombia, \\ orlandocastellanos7@hotmail.com
}

\begin{abstract}
Cómo referenciar / How to cite
Grajales-Gaviria, D.A. y Castellanos-Polo, O.C. (2018). Evaluación del control interno en el proceso de tesorería de las pequeñas y medianas empresas de Medellín. Revista CEA, 4(7), 67-83. https://doi.org/10.22430/24223182.760
\end{abstract}

Recibido: 31 de agosto de 2017

Aceptado: 6 de octubre de 2017

\section{Resumen}

Al evaluar la empresa por áreas de trabajo, se identifica que el departamento más susceptible a fraudes es el de Tesorería, por esta razón, se propone evaluar la estructura del control interno con énfasis al ciclo transaccional del proceso de tesorería en las pequeñas y medianas empresas por medio de una muestra representativa en el municipio de Medellín.

Esta revisión permitirá identificar debilidades en los procesos y brindar opciones de mejora con el fin de garantizar la eficacia, eficiencia, efectividad y economía en la gestión institucional.

El alcance de esta investigación está basado en los componentes del modelo de control interno COSO, los cuáles serán el enfoque modular para la revisión del proceso de tesorería; Se revisarán: El proceso de cuentas por pagar, el proceso de cuentas por cobrar, el proceso de recaudo de efectivo y el proceso de pagos de obligaciones.

El resultado de esta investigación permitirá alcanzar el objetivo de identificar las principales falencias en el proceso de tesorería en cuanto a los mecanismos de control interno que las pequeñas y medianas empresas de Medellín tienen estipulados.

Palabras clave: proceso, control interno, riesgos, ciclo de tesorería, auditoria.

\section{Abstract}

An evaluation of the departments of companies reveals that the most susceptible to fraud is corporate treasury. For that reason, this work proposes to evaluate the internal control structure, specially the transaction cycle of the treasury process at small and 
medium-sized enterprises by means of a representative sample in the Municipality of Medellín. This review will enable to identify weaknesses in the processes and provide improvement alternatives to ensure efficacy, efficiency, effectiveness and economy in corporate management.

The scope of this research is based on the components of the COSO internal control model, which will be the modular approach for the assessment of the treasury process. This study analyses several processes: Accounts payable, Accounts receivable, Cash collection, and Obligation payment.

The results will enable to identify the main deficiencies in the treasury process regarding internal control mechanisms established by small and medium-sized enterprises in Medellín.

Keywords: process, internal control, risks, treasury cycle, auditing.

\section{INTRODUCCIÓN}

El control interno es un proceso ejecutado en cada una de las áreas de trabajo de la organización, se evidencia en los procedimientos establecidos, en las políticas, en los mecanismos de control y en la actitud de todo el personal. El control interno comprende el plan de organización y el conjunto de métodos y medidas adoptadas dentro de una entidad para salvaguardar sus recursos. (Capote, 2000). A nivel global, se han identificado vacíos en este proceso que llevaron a la formulación de modelos de control, buscando salvaguardar los activos y el cumplimiento de las metas organizacionales, para evitar fraudes organizacionales 0 desviaciones en el cumplimiento de los objetivos; los modelos de control más reconocidos en Latinoamérica son: el modelo
COSO, COBIT, COCO, CADBURY y el MECI. Estas herramientas se encuentran en constante actualización y mejora para el cumplimiento de objetivos a nivel organizacional. Otra definición aceptada de control interno es: el plan de organización de todos los métodos y procedimientos que son relativos y que están directamente relacionados principalmente con la salvaguarda de activos. (Cartategui, 2009) Sin embargo, aunque existen estos modelos que ayudan a la institución como mecanismo para disminuir la materialización de riesgos, se puede evidenciar que en la actualidad se siguen presentando vacíos que permiten la ocurrencia de situaciones como el fraude.

El fraude en las compañías se ha observado con mayor frecuencia en los últimos años en el departamento de Tesorería y las áreas que se encuentran en constante relación con esta dependencia, la identificación de esta situación genera la necesidad de evaluar el sistema de control interno en el proceso de tesorería, evaluando en este, los controles existentes en cada una de las compañías seleccionadas con el fin de determinar los riesgos inherentes que se puedan presentar en estos. Para realizar el análisis se toma como población las pequeñas y medianas empresas del municipio de Medellín, en las cuales se dará un diagnóstico del manejo que se da al control interno en este departamento brindando recomendaciones de acuerdo con lo encontrado y buscando que la adecuada estructuración de procesos institucionales optimice en sus mecanismos de control y con esto redunde en la óptima gestión de la compañía.

La adecuada estructuración de procesos institucionales se convierte en un mecanismo de control que genera confianza y protección a los recursos de los inversionistas, sin embargo, las debilidades de dichos procesos también generan riesgos como el fraude, el robo y en general pérdida de recursos económicos o de imagen para las compañías. 
La metodología utilizada para el desarrollo del presente trabajo es el enfoque cuantitativo descriptivo con un método de estudio fenomenológico, debido a que busca la obtención de información de los distintos procesos y mecanismos de control desarrollados en el proceso de tesorería de las pequeñas y medianas empresas ubicadas en la ciudad de Medellín, para proponer recomendaciones que permitan la consolidación de la calidad y el logro de los objetivos en las organizaciones.

\section{MARCO TEÓRICO}

\section{El control interno}

El crecimiento y desarrollo social desde el siglo XX al siglo XXI han obligado a las organizaciones en general a desarrollar mecanismos de control, que permitan de una forma organizada y fragmentada, entregar o suministrar información de las acciones realizadas en los diferentes procesos misionales, sin embargo, estos procesos necesitan ser revisados desde su lógica de funcionamiento, se puede encontrar, que la realidad de funcionamiento no garantiza el control de los activos empresariales y que dichos procesos deben tener un mayor nivel de control, estos elementos son abordados desde el control interno como herramienta de gestión a los procesos empresariales.

El mejoramiento del sistema de control interno puede plantearse como una necesidad de infraestructura tecnológica "IT" que puede ser considerada como una herramienta válida para la estructuración del control interno organizacional. Al respecto (Vélez \& Duque, 2017) expresan: «Si bien, un mismo problema de IT puede presentarse en varias compañías, no existe la disposición ni el tiempo para que se comparta esta información y se construya una base de conocimiento que les permita mejorar los tiempos de respuesta y la presentación de soluciones oportunas.» Este problema es un factor clave en las pymes que carecen de recursos para invertir en infraestructura tecnológica que les permita estructurar un mejor sistema de control interno, sin embargo, el control interno no se limita solo a la IT, es toda una estructura organizacional que permite a los empresarios tener tranquilidad y certeza de que sus recursos se encuentran bien administrados en las sociedades que han invertido.

Al respecto de control interno se han generado diversos modelos a nivel global dentro de los cuales se destacan por su nivel de acogida el modelo COSO, CADBURY y en Colombia el modelo $\mathrm{MECl}$. (Departamento Administrativo de la función Pública, 2014)

El control interno según el modelo Cadbury presenta la siguiente perspectiva, como lo manifiesta (Argente, 2012).

El control interno está formado por un conjunto de derechos y normas que comprenden dos elementos básicos: por un lado, la estructura que va a determinar cuál va a ser la distribución de derechos y responsabilidades entre el cuerpo directivo, los accionistas y el cuerpo directivo de administración; $y$, por otro, los procedimientos necesarios para la toma de decisión en cuestiones estratégicas y corporativas.

Definición de Control Interno según (COSO, 2016):

El control interno es definido como un proceso llevado a cabo por el consejo de administración, la dirección y el resto del personal de una entidad, diseñado con el objeto de proporcionar un grado de seguridad razonable en cuanto a la consecución de objetivos relaciona-dos 
con las operaciones, la información y el cumplimiento.

Como marco de referencia de esta investigación, se utiliza el modelo coso del cual se toman sus componentes y criterios de percepción del control interno.

El control interno refleja ciertos conceptos fundamentales como: el proceso se entiende como una serie de actividades que van todas enmarcadas hacia la obtención de un fin, se entiende siempre que un adecuado sistema de control interno no es garantía absoluta del logro de objetivos, pero sí es una herramienta esencial para la adecuada toma de decisiones por parte de los altos directivos, el control interno organizacional debe ser flexible $y$ permitir ser modificado ante los cambios presentados en el entorno organizacional y las nuevas realidades que hacen que un sistema válido en determinadas circunstancias pierda total nivel de confianza por ejemplo con los cambios tecnológicos.

Como expresa (Ortiz, Esquivel \& Hernández, 2016) "las empresas, para sobrevivir en el entorno competitivo actual deben centrarse en la consecución de una ventaja competitiva sostenible, basada en el conocimiento, que las diferencie de su competencia". Este aspecto se logra con una adecuada estructura de control interno que le permite a la sociedad tener claridad de sus fortalezas y aspectos débiles para mejorar y afrontar el mercado.

El control interno según lo expresa (Lara, 2007), se puede clasificar en los siguientes momentos:

- Revisión del Sistema de Control Interno: consiste en la revisión del sistema con objeto de conocer y comprender los métodos establecidos por la entidad. La revisión del sistema es principalmente un proceso de obtención de información respecto a la organización y a los procesos establecidos, con objeto que sirva como base para las pruebas de cumplimiento y para la evaluación del sistema.

- Evaluación del Sistema de Control Interno: pruebas de Cumplimiento, consiste en la realización de pruebas de cumplimiento para obtener unas seguridades razonables de que los controles se encuentran en uso y que están operando tal como se diseñaron.

Estas etapas denotan la necesidad organizacional de revisar y realizar evaluaciones a los procesos organizacionales para garantizar el adecuado funcionamiento del sistema de control interno y con garantizar la salvaguarda de los activos de los inversionistas en las sociedades.

\section{El ciclo de tesorería}

El ciclo de tesorería según Estupiñán (2015) comprende aquellas funciones relativas a los fondos de capital, por lo tanto, se inicia considerando las necesidades de efectivo, distribución y aplicación del disponible, culminándose con su retorno a las funciones que lo originaron, como inversionistas, acreedores.

La tesorería, como proceso organizacional, presenta elementos inherentes como son: la custodia de efectivo, las conciliaciones de cuentas, las relaciones con proveedores, los ingresos y pagos en general estos procesos están vinculados a otros componentes del movimiento del dinero en la organización, como son los recaudos y la compra de inventarios o activos. 


\section{METODOLOGÍA}

La población para el desarrollo del trabajo son las pequeñas y medianas empresas ubicadas en el municipio de Medellín, que para el año 2016, según el informe de estructura empresarial emitido por (Cámara de Comercio de Medellín para Antioquia, 2016) de acuerdo con el tamaño de empresa es: Pequeña empresa 9879 y Mediana empresa 2575. De esta población, se tomó una muestra de 373 empresas con un margen de error del $5 \%$ y un nivel de confianza del $95 \%$, a las que se les realizó inicialmente una encuesta como lista de chequeo para evaluar el proceso de tesorería y el manejo que en este se da del control interno, partiendo del modelo de control COSO; dentro del proceso de tesorería se evaluaron los procesos y transacciones que se realizan en esta área, los cuales son: procesos de cuentas por pagar, procesos de cuentas por cobrar, procesos de recaudo de efectivo, y procesos de pago de obligaciones.

\section{Instrumentos de medición y técnicas}

La metodología utilizada para el desarrollo del presente trabajo fue un enfoque cuantitativo descriptivo, con un método de estudio fenomenológico, debido a que busca la obtención de información de los distintos procesos y mecanismos de control desarrollados en la tesorería de las pequeñas y medianas empresas ubicadas en la ciudad de Medellín, para proponer recomendaciones que permitan la consolidación de la calidad y el logro de los objetivos en las organizaciones.

Los materiales utilizados fueron la lista de chequeo realizada a los directivos de 373 empresas tomadas como muestra, y posteriormente de esta muestra, se utilizaron 20 empresas después de solicitar acceso a la información física de las 373 compañías de la muestra inicial y ante la imposibilidad de acceder físicamente al proceso de todas, esta situación se presentó principalmente por políticas de las sociedades o por falta de respuesta a la solicitud de acceso a los procesos.

La escala de valoración para la medición del riesgo en los procesos de tesorería fue de alto, medio y bajo, con una medición porcentual de los siguientes rangos

Alto: $71 \%$ hasta el $100 \%$

Medio: $34 \%$ hasta el $70 \%$

Bajo: $0 \%$ hasta el $33 \%$

Este grado de valoración se determina de forma propia, partiendo de los lineamientos propuestos por el modelo de control interno COSO ERM.

\section{La recolección de datos}

Lista de chequeo evaluación de componentes de control

La información de las pequeñas y medianas empresas se obtuvo con la lista de chequeo estructurada con preguntas de elaboración propia, con base en cada uno de los componentes de control interno del modelo COSO, que permitió evaluar el manejo de los componentes de control interno en el proceso de tesorería de este tipo de empresas, además de la utilización de las técnicas de auditoría en las empresas seleccionadas para revisar sus procesos de forma física. Esta metodología permitió la obtención de evidencias suficientes sobre las cuales emitir una opinión respecto al proceso auditado. Las actividades realizadas en las 20 empresas seleccionadas para la muestra física fueron: la consulta, observación, inspección, y revisión de comprobantes.

A continuación, se muestra los resultados de la lista de chequeo aplicada a cada uno de los componentes de control interno. (ver Tablas 17): 
Componente 1 -Ambiente de control

Es la base sobre la que se estructura todo el sistema de control organizacional, este permite identificar elementos esenciales de control como son: los valores, el recurso humano, la forma de direcciona organizacional y el autocontrol.

Se evidencia en la evaluación de este componente un nivel de riesgo promedio de 56 $\%$, identificado como un nivel de riesgo medio para las pequeñas y medianas empresas de Medellín. Se muestra que elementos del componente, como valores, moral e indicadores de medición, no existen en este tipo de organizaciones.

\section{Componente 2 - Evaluación de riesgos}

La revisión de los riesgos organizacionales es una práctica que le permite a la compañía determinar los factores que pueden generar la materialización de riesgos, esto se logra por medio de identificación clara de objetivos, del análisis de impactos organizacionales, por la materialización del riesgo y de las acciones que permitirán tanto prevenir como disminuir la probabilidad de ocurrencia del riesgo.

Se evidencia un nivel de riesgo alto en este componente, lo cual muestra que las pequeñas y medianas empresas en Medellín no tienen presente la evaluación de riesgos en su concepción de control interno; además, se muestra que elementos como la administración del riesgo ni siquiera se perciben como importantes para las compañías encuestadas.

\section{Componente 3 -Actividades de control}

Son los mecanismos utilizados por la gerencia, y en general por todos los colaboradores de la compañía, como herramientas de control que permitan evitar la materialización de riesgos Dentro de estas herramientas se pueden identificar: la adecuada asignación de responsabilidades, las políticas y normas internas y los mecanismos que permiten dar cumplimiento a las normas que en general debe cumplir la organización.

Las actividades de control existen en las pequeñas y medianas empresas, pero estas se vuelven estáticas, inamovibles, como se evidencia en la tendencia de preguntas, como: ¿Las políticas son validadas periódicamente? Encontrando que el $100 \%$ de las empresas dicen no validarlas. Al igual que la no existencia de indicadores de gestión y otros elementos que generan un adecuado sistema de control de la información y protección de los activos organizacionales.

\section{Componente 4-Información y comunicación}

Tan importante como las actividades de control, la información organizacional debe permitir a la compañía que las políticas, normas internas procesos y actividades organizacionales sean oportunamente entregadas a los responsables, de la misma forma con los terceros que tienen vínculo con la organización, como son acreedores, clientes, entidades bancarias y todos aquellos a los que la información financiera de la empresa pueda afectar en alguna medida.

Este componente presenta un riesgo medio evidenciando que existe comunicación en las empresas, aunque esta presenta debilidades, como son la no existencia de políticas para el manejo de la información y la percepción general del sistema de información el cual se encuentra con vacíos de fondo en las pequeñas y medianas empresas encuestadas. 
Tabla 1. Componente 1 - Integridad y valores éticos

Table 1. Component 1- Integrity and ethical values

\begin{tabular}{|c|c|c|c|}
\hline & INTEGRIDAD Y VALORES ÉTICOS & Valor & Riesgo \\
\hline 1.1 & ¿Conoce el código de conducta y ética? & 100 & Alto \\
\hline 1.2 & $\begin{array}{l}\text { ¿Contamos con una cultura corporativa que incorpora valores, y se } \\
\text { evalúa? }\end{array}$ & 50 & Medio \\
\hline 1.3 & $\begin{array}{l}\text { ¿Considera que han sido suficientemente difundidos los valores } \\
\text { institucionales? }\end{array}$ & 30 & Bajo \\
\hline 1.4 & ¿Existe un compromiso con estos valores a nivel personal y laboral? & 30 & Bajo \\
\hline 1.5 & $\begin{array}{l}\text { ¿Existen mecanismos corporativos que inculquen de forma permanente } \\
\text { los valores deseados en nuestro recurso humano? }\end{array}$ & 30 & Bajo \\
\hline 1.6 & ¿Se realizan mediciones de valores en el plano personal? & 100 & Alto \\
\hline 1.7 & ¿Se han definido indicadores de valores éticos y morales? & 100 & Alto \\
\hline 1.8 & $\begin{array}{l}\text { ¿Se han definido indicadores de valores éticos y morales deseados en la } \\
\text { organización? }\end{array}$ & 100 & Alto \\
\hline 1.9 & $\begin{array}{l}\text { ¿Se han definido indicadores de valores éticos y morales deseados en el } \\
\text { recurso humano? }\end{array}$ & 100 & Alto \\
\hline 1.10 & $\begin{array}{l}\text { ¿Se puede tolerar recurso humano que no posee los valores } \\
\text { corporativos? }\end{array}$ & 30 & Bajo \\
\hline 1.11 & ¿Contamos con una cultura corporativa que incorpora valores? & 30 & Bajo \\
\hline 1.12 & ¿Se realizan mediciones anuales de integridad? & 100 & Alto \\
\hline 1.13 & ¿Existen indicadores de integridad administrativa? & 100 & Alto \\
\hline 1.14 & ¿Se evalúan las técnicas administrativas para la consecución de metas? & 100 & Alto \\
\hline \multirow[t]{2}{*}{1.15} & $\begin{array}{l}\text { ¿Contamos con un estándar de valores de los líderes de nuestra } \\
\text { organización? }\end{array}$ & 30 & Bajo \\
\hline & Total Promedio & 77 & Alto \\
\hline
\end{tabular}

Fuente: elaboración propia.

Tabla 2. Componente 1 - Recurso humano

Table 2. Component 1 - Human resources.

\begin{tabular}{|c|c|c|c|}
\hline 2. & HABILIDADES DEL RECURSO HUMANO & Valor & Riesgo \\
\hline 2.1 & $\begin{array}{l}\text { ¿Existen mediciones de las competencias que reflejen sus conocimientos } \\
\text { y habilidades en el cargo, y se evalúan periódicamente? }\end{array}$ & 100 & Alto \\
\hline 2.2 & ¿Existen planes de promoción y desarrollo profesional dentro del área? & 100 & Alto \\
\hline 2.3 & $\begin{array}{l}\text { ¿Los incentivos y reconocimientos están acordes con las } \\
\text { responsabilidades y competencias del cargo? }\end{array}$ & 100 & Alto \\
\hline 2.4 & ¿Los incentivos dependen exclusivamente de resultados? & 100 & Alto \\
\hline 2.5 & $\begin{array}{l}\text { ¿Existe una política o proceso que trace tanto los compromisos, como } \\
\text { los incentivos que tendrán nuestros colaboradores durante la } \\
\text { permanencia en la organización? }\end{array}$ & 100 & Alto \\
\hline 2.6 & $\begin{array}{l}\text { ¿Existe un plan de desarrollo personal y profesional de nuestro recurso } \\
\text { humano dentro de la organización? }\end{array}$ & 100 & Alto \\
\hline \multirow[t]{2}{*}{2.7} & $\begin{array}{l}\text { ¿Los informes de Auditoría son apoyo para la toma de decisiones } \\
\text { administrativas y legales en casos de conductas impropias? }\end{array}$ & 100 & Alto \\
\hline & Total Promedio & 100 & Alto \\
\hline
\end{tabular}


Tabla 3. Componente 1 - Estilo de operación y estructura organizacional

Table 3. Component 1 - Operational style and organizational structure

\begin{tabular}{|c|c|c|c|}
\hline 3. & ESTILO DE OPERACIÓN Y ESTRUCTURA ORGANIZACIONAL & Valor & Riesgo \\
\hline 3.1 & ¿Las acciones administrativas fortalecen el sistema de control interno? & 30 & Bajo \\
\hline 3.2 & ¿La compañía apoya el sistema de control interno en políticas operativas? & 30 & Bajo \\
\hline 3.3 & $\begin{array}{l}\text { ¿Sus acciones individuales han sido respaldadas por los demás } \\
\text { compañeros de su dependencia? }\end{array}$ & 30 & Bajo \\
\hline 3.4 & $\begin{array}{l}\text { ¿Usted le da importancia a la cultura del control en el desarrollo de sus } \\
\text { funciones? }\end{array}$ & 100 & Alto \\
\hline 3.5 & $\begin{array}{l}\text { ¿Están identificados todos los componentes del sistema de Control } \\
\text { interno en la corporación? }\end{array}$ & 30 & Bajo \\
\hline 3.6 & $\begin{array}{l}\text { ¿La alta gerencia conoce los entes reguladores y normas que impactan en } \\
\text { la operación del negocio? }\end{array}$ & 30 & Bajo \\
\hline 3.7 & $\begin{array}{l}\text { ¿El sistema de control interno corporativo cumple con las exigencias } \\
\text { externas y asegura el cumplimiento de los objetivos del negocio? }\end{array}$ & 30 & Bajo \\
\hline 3.8 & $\begin{array}{l}\text { ¿EL Director emite las pautas para los factores de control interno } \\
\text { corporativo? } \\
\text { (ambiente de control, valoración del riesgo, actividades de control, } \\
\text { información y comunicación, monitoreo) }\end{array}$ & 100 & Alto \\
\hline 3.9 & $\begin{array}{l}\text { ¿Todas La unidades estratégicas de negocio tiene implementado el } \\
\text { sistema de control interno acorde con sus objetivos específicos? }\end{array}$ & 100 & Alto \\
\hline 3.10 & $\begin{array}{l}\text { ¿Se monitorea y evalúa el cumplimiento de las políticas operativas } \\
\text { corporativas? }\end{array}$ & 100 & Alto \\
\hline 3.11 & ¿Las acciones administrativas fortalecen el sistema de control interno? & 30 & Bajo \\
\hline 3.12 & $\begin{array}{l}\text { ¿El sistema de información financiera garantiza la integridad, } \\
\text { confiabilidad, disponibilidad, veracidad, efectividad y eficacia de las } \\
\text { operaciones y cumplimiento con leyes y regulaciones? }\end{array}$ & 30 & Bajo \\
\hline 3.13 & $\begin{array}{l}\text { ¿El sistema de información financiera cuenta con una herramienta que } \\
\text { permita ser auditada? }\end{array}$ & 30 & Bajo \\
\hline 3.14 & $\begin{array}{l}\text { ¿El sistema de control interno corporativo está avalado por Auditoría } \\
\text { Interna? }\end{array}$ & 30 & Bajo \\
\hline 3.15 & $\begin{array}{l}\text { ¿La estructura organizacional satisface los requerimientos de la } \\
\text { organización? }\end{array}$ & 30 & Bajo \\
\hline 3.16 & $\begin{array}{l}\text { ¿Está identificado el flujo de la información dentro de la estructura } \\
\text { organizacional? }\end{array}$ & 30 & Bajo \\
\hline 3.17 & $\begin{array}{l}\text { ¿Todos los cargos poseen guías ocupacionales, donde están especificadas } \\
\text { sus funciones? }\end{array}$ & 30 & Bajo \\
\hline 3.18 & $\begin{array}{l}\text { ¿La alta gerencia tiene definidas y conoce sus responsabilidades en los } \\
\text { macroprosesos del negocio? }\end{array}$ & 30 & Bajo \\
\hline \multirow[t]{2}{*}{3.19} & $\begin{array}{l}\text { ¿La gerencia ha segregado las funciones y responsabilidades de cada } \\
\text { proceso de la organización? }\end{array}$ & 30 & Bajo \\
\hline & Total Promedio & 45 & Medio \\
\hline
\end{tabular}




\begin{tabular}{|c|c|c|}
\hline 4. VALORACIÓN DE AUTORIDAD Y RESPONSABILIDAD & Valor & Riesgo \\
\hline 4.1 ¿Considera que todos los funcionarios conocen los procesos del área? & 30 & Bajo \\
\hline $\begin{array}{l}\text { 4.2 ¿Considera usted que la forma como es asignada la responsabilidad es } \\
\text { adecuada? }\end{array}$ & 30 & Bajo \\
\hline 4.3 ¿El diálogo entre jefes y funcionarios garantiza resultados satisfactorios? & 30 & Bajo \\
\hline $\begin{array}{l}\text { 4.4 ¿La responsabilidad del cumplimiento de los objetivos organizacionales se } \\
\text { encuentra asignada a los dueños de los negocios? }\end{array}$ & 30 & Bajo \\
\hline $\begin{array}{l}4.5 \text { ¿Las políticas, normas y procedimientos aseguran el cumplimiento de les } \\
\text { leyes y normas que rigen el negocio? }\end{array}$ & 30 & Bajo \\
\hline 4.6 ¿La operación del negocio está documentada? & 30 & Bajo \\
\hline $\begin{array}{l}4.7 \text { ¿Existen planes de contingencia que aseguren la continuidad de las } \\
\text { operaciones del negocio? }\end{array}$ & 30 & Bajo \\
\hline Total Promedio & 30 & Bajo \\
\hline 5. AUTOCONTROL & Valor & Riesgo \\
\hline 5.1 ¿Tiene claridad del concepto de autocontrol? & 30 & Bajo \\
\hline 5.2 ¿Ha interiorizado el tema de autocontrol? & 30 & Bajo \\
\hline $\begin{array}{l}\text { La aplicación del autocontrol le ha permitido optimizar el desarrollo de sus } \\
\text { tareas y actividades }\end{array}$ & 30 & Bajo \\
\hline Total Promedio & 30 & Bajo \\
\hline TOTAL COMPONENTE DE CONTROL & 56 & Medio \\
\hline
\end{tabular}

Fuente: elaboración propia.

Tabla 4. Componente 2 - Objetivos globales del área y a nivel de actividad Table 4. Component 2 - Global and activity-level objectives of the department

\begin{tabular}{|c|c|c|c|}
\hline & OBJETIVOS GLOBALES DEL ÁREA Y A NIVEL DE ACTIVIDAD & Valor & Riesgo \\
\hline \multirow{2}{*}{\multicolumn{4}{|c|}{$\frac{1.1 \text { ¿Están identificados los objetivos comunes del área? }}{1}$}} \\
\hline & & & \\
\hline & $\begin{array}{l}\text {. ¿Las estrategias operativas son definidas y difundidas dentro de la } \\
2 \text { dependencia? }\end{array}$ & 40 & Medio \\
\hline 1.3 & $\begin{array}{l}\text { ¿Los objetivos del área satisfacen las necesidades y atención a la } \\
\text { comunidad? }\end{array}$ & 40 & Medio \\
\hline & Total Promedio & 40 & Medio \\
\hline 2. & ADMINISTRACIÓN DEL RIESGO & Valor & Riesgo \\
\hline 2.1 & $\begin{array}{l}\text { ¿Conoce usted los factores de riesgo que hay en la realización de sus } \\
\text { actividades? }\end{array}$ & 100 & Alto \\
\hline 2.2 & $\begin{array}{l}\text { ¿Se le da el suficiente nivel de importancia a los riesgos a que está sometida } \\
\text { este departamento? }\end{array}$ & 100 & Alto \\
\hline 2.3 & ¿Ha tomado medidas para que estos factores sean controlables? & 100 & Alto \\
\hline
\end{tabular}




\begin{tabular}{|c|c|c|c|}
\hline 2.4 & $\begin{array}{l}\text { ¿Están identificados los riesgos internos y externos asociados a los objetivos } \\
\text { organizacionales? }\end{array}$ & 100 & Alto \\
\hline 2.5 & $\begin{array}{l}\text { ¿Existe una metodología que valore los riesgos en forma dinámica e } \\
\text { interactiva? }\end{array}$ & 100 & Alto \\
\hline 2.6 & $\begin{array}{l}\text { ¿Se monitorean los riesgos en forma periódica acorde con las unidades } \\
\text { estratégicas de negocio? }\end{array}$ & 100 & Alto \\
\hline 2.7 & ¿La metodología pondera los riesgos de forma cualitativa y cuantitativa? & 100 & Alto \\
\hline 2.8 & $\begin{array}{l}\text { ¿Están incluidos los factores internos y externos que impactan la ocurrencia } \\
\text { de los riesgos identificados? }\end{array}$ & 100 & Alto \\
\hline & Total Promedio & 100,00 & Alto \\
\hline 3. & MANEJO DEL RIESGO & Valor & Riesgo \\
\hline 3.1 & ¿Tiene el área algún plan de manejo de factores de riesgo? & 100 & Alto \\
\hline 3.2 & ¿Es este plan es lo suficientemente comprendido para su desarrollo? & 100 & Alto \\
\hline 3.3 & ¿Este plan está integrado a las acciones diarias realizadas en el área? & 100 & Alto \\
\hline 3.4 & ¿Usted tiene conocimiento de la ejecución de este plan? & 100 & Alto \\
\hline 3.5 & $\begin{array}{l}\text { ¿Se monitorear los riesgos en forma periódica de acuerdo con el nivel de } \\
\text { operaciones? }\end{array}$ & 100 & Alto \\
\hline & Total Promedio & 100 & Alto \\
\hline 4. & ADMINISTRACIÓN DEL CAMBIO & Valor & Riesgo \\
\hline 4.1 & $\begin{array}{l}\text { Los planes para guiar los esfuerzos hacia el logro del objetivo del área son } \\
\text { establecidos y comunicados }\end{array}$ & 30 & Bajo \\
\hline 4.2 & $\begin{array}{l}\text { ¿Considera que se toman medidas apropiadas con relación a los cambios y } \\
\text { externos en su área? }\end{array}$ & 30 & Bajo \\
\hline 4.3 & $\begin{array}{l}\text { ¿Las políticas diseñadas para apoyar el logro de los objetivos } \\
\text { organizacionales y la administración de sus riesgos, son establecidas, } \\
\text { comunicadas y practicadas de manera que todo el equipo humano } \\
\text { entienda lo que se espera del? }\end{array}$ & 74 & Alto \\
\hline 4.4 & $\begin{array}{l}\text { ¿Cómo identifica los cambios internos y externos que tendrán impacto en } \\
\text { los planes estratégicos? }\end{array}$ & 34 & Medio \\
\hline 4.5 & $\begin{array}{l}\text { ¿Ha llevado a cabo un análisis de sensibilidad para identificar los efectos de } \\
\text { los cambios en proyecciones estratégicas? }\end{array}$ & 50 & Medio \\
\hline 4.6 & $\begin{array}{l}\text { ¿La situación actual está alineada con las proyecciones esperadas cuando } \\
\text { fueron aprobados los planes? }\end{array}$ & 34 & Medio \\
\hline 4.7 & ¿Aún son vigentes estas proyecciones estratégicas? & 34 & Medio \\
\hline 4.8 & $\begin{array}{l}\text { ¿Cómo puede la Junta ayudar a identificar riesgos que la estructura de } \\
\text { riesgos no permita ver? }\end{array}$ & 100 & Alto \\
\hline 4.9 & $\begin{array}{l}\text { ¿Cuáles son las ventajas y desventajas de implementar los cambios } \\
\text { identificados? }\end{array}$ & 34 & Medio \\
\hline 4.10 & $\begin{array}{l}\text { ¿Fueron consideradas varias alternativas y se selecciona la que está } \\
\text { alineada a los objetivos del negocio? }\end{array}$ & 34 & Medio \\
\hline & Total Promedio & 45,4 & Medio \\
\hline & TOTAL COMPONENTE EVALUACIÓN DE RIESGOS & 71,35 & Alto \\
\hline
\end{tabular}

Tabla 5. Componente 3 - Funciones directas o actividades administrativas 
Table 5. Component 3 - Direct responsibilities or administrative activities

\begin{tabular}{|c|c|c|c|}
\hline & FUNCIONES DIRECTAS O ACTIVIDADES ADMINISTRATIVAS & Valor & Riesgo \\
\hline 1.1 & $\begin{array}{l}\text { ¿Se cuenta con una estrategia de comunicación efectiva hacia los altos } \\
\text { niveles de la organización? }\end{array}$ & 34 & Medio \\
\hline 1.2 & $\begin{array}{l}\text { ¿El nivel directivo promueve y ejemplariza el mejoramiento del sistema } \\
\text { de control interno? }\end{array}$ & 34 & Medio \\
\hline & Total Promedio & 34 & Medio \\
\hline 1 & Evaluación de alto nivel & Valor & Riesgo \\
\hline 1.1 & ¿Están definidos los indicadores de gestión organizacional? & 100 & Alto \\
\hline 1.2 & ¿Se hace monitoreo a estos indicadores? & 100 & Alto \\
\hline 1.3 & $\begin{array}{l}\text { ¿Se realizan informes con el análisis de los principales indicadores de } \\
\text { gestión? }\end{array}$ & 100 & Alto \\
\hline & Total Promedio & 100 & Alto \\
\hline 2. & NORMAS Y DISPOSICIONES INTERNAS & Valor & Riesgo \\
\hline 2.1 & $\begin{array}{l}\text { ¿Existen normas y disposiciones sobre control interno acordes con las } \\
\text { necesidades institucionales? }\end{array}$ & 34 & Medio \\
\hline 2.2 & ¿Existe un medio de difusión oportuno y efectivo de estas normas? & 34 & Medio \\
\hline 2.3 & Existe un medio para evaluar su grado de aplicación? & 100 & Alto \\
\hline & Total Promedio & 56,00 & Medio \\
\hline 3. & PROCESAMIENTO DE LA INFORMACIÓN & Valor & Riesgo \\
\hline 3.1 & $\begin{array}{l}\text { ¿Existen políticas y procedimientos definidos para el ingreso de la } \\
\text { información contable? }\end{array}$ & 30 & Bajo \\
\hline 3.2 & $\begin{array}{l}\text { ¿Se cuenta con los recursos apropiados para el procesamiento de la } \\
\text { información? }\end{array}$ & 74 & Alto \\
\hline 3.3 & $\begin{array}{l}\text { ¿Se asegura y verifica la adecuada segregación de funciones en el } \\
\text { procesamiento de la información? }\end{array}$ & 34 & Medio \\
\hline 3.4 & ¿Existen políticas y procedimientos para atender los requerimientos de: & 100 & Alto \\
\hline 3.5 & ¿Estructura de datos? & 100 & Alto \\
\hline 3.6 & ¿Conectividad? & 100 & Alto \\
\hline 3.7 & ¿Hardware? & 100 & Alto \\
\hline 3.8 & ¿Software? & 100 & Alto \\
\hline & Total Promedio & 79,75 & Alto \\
\hline 4. & POLÍTICAS Y PROCEDIMIENTOS & Valor & Riesgo \\
\hline 4.1 & ¿Las políticas institucionales son conocidas? & 30 & Bajo \\
\hline 4.2 & Dichas políticas se cumplen y son validadas periódicamente? & 100 & Alto \\
\hline 4.3 & $\begin{array}{l}\text { ¿Los procedimientos institucionales son conocidos y aplicados en todos } \\
\text { los niveles de la organización? }\end{array}$ & 30 & Bajo \\
\hline 4.4 & $\begin{array}{l}\text { ¿Contamos con mecanismos de monitoreo para los procesos y } \\
\text { procedimientos en las Unidades Estratégicas de Negocio (UEN's)? }\end{array}$ & 100 & Alto \\
\hline 4.5 & ¿Las políticas corporativas son conocidas? & 34 & Medio \\
\hline
\end{tabular}




\begin{tabular}{llcr}
\hline 4.6 & ¿Se cumplen estas políticas? & 30 & Bajo \\
\hline 4.7 ¿Las políticas son validadas periódicamente? & 100 & Alto \\
\hline $4.8 \begin{array}{l}\text { ¿Los cambios a las políticas, normas y procedimientos son avalados por } \\
\text { los dueños de los negocios? }\end{array}$ & 30 & Bajo \\
\hline 4.9 ¿Los procedimientos corporativos son conocidos? & 30 & Bajo \\
\hline 4.10 & 30 & Bajo \\
\hline ¿Los procedimientos corporativos son aplicados? & 51,40 & Medio \\
\hline Total Promedio & & \\
\hline TOTAL COMPONENTE ACTIVIDADES DE CONTROL & 64,23 & Medio \\
\hline
\end{tabular}

Fuente: elaboración propia.

Tabla 6. Componente 4 - Información organizacional

Table 6. Component 4 - Organizational information

\begin{tabular}{|c|c|c|c|}
\hline 1. & INFORMACIÓN & Valor & Riesgo \\
\hline 1.1 & $\begin{array}{l}\text { ¿Considera que se encuentra capacitado en la utilización de los sistemas } \\
\text { de información? }\end{array}$ & 100 & Alto \\
\hline 1.2 & $\begin{array}{l}\text { ¿El suministro de información a la gente clave se da con tiempo suficiente } \\
\text { y bien detallada de manera que ellos puedan cumplir sus } \\
\text { responsabilidades? }\end{array}$ & 100 & Alto \\
\hline 1.3 & $\begin{array}{l}\text { ¿Considera que se realiza una evaluación y seguimiento al procesamiento } \\
\text { de datos e informes? }\end{array}$ & 100 & Alto \\
\hline 1.4 & $\begin{array}{l}\text { ¿Se tienen definidas las políticas y procedimientos de privacidad, } \\
\text { mantenimiento y flujo de datos del software aplicativo? }\end{array}$ & 100 & Alto \\
\hline 1.5 & $\begin{array}{l}\text { ¿Existe un plan estratégico de tecnología de información a corto y largo } \\
\text { plazo? }\end{array}$ & 100 & Alto \\
\hline 1.6 & $\begin{array}{l}\text { ¿Existe un modelo de arquitectura de información del plan de TI y está } \\
\text { documentado? }\end{array}$ & 100 & Alto \\
\hline 1.7 & $\begin{array}{l}\text { ¿xisten políticas, procedimientos y estándares para elaborar y mantener } \\
\text { actualizado el plan de infraestructura tecnológica del plan? }\end{array}$ & 100 & Alto \\
\hline 1.8 & ¿Existe un presupuesto de inversiones de TI alineado con el plan? & 100 & Alto \\
\hline 1.9 & $\begin{array}{l}\text { ¿Se ubica a cada funcionario donde es apropiado de acuerdo con su } \\
\text { grado de conocimientos, experiencia, habilidades y acreditación } \\
\text { académica?. }\end{array}$ & 100 & Alto \\
\hline 1.10 & $\begin{array}{l}\text { ¿Se dispone de bitácoras para el registro de incumplimientos de los } \\
\text { requerimientos legales y tecnológicos? }\end{array}$ & 100 & Alto \\
\hline 1.11 & $\begin{array}{l}\text { ¿Existe una metodología para la administración de proyectos tecnológicos } \\
\text { en la organización? }\end{array}$ & 100 & Alto \\
\hline 1.12 & $\begin{array}{l}\text { ¿Existen políticas y procedimientos que permitan definir y determinar los } \\
\text { requerimientos de usuarios? }\end{array}$ & 100 & Alto \\
\hline 1.13 & $\begin{array}{l}\text { ¿Existen responsables por la administración de los cambios o } \\
\text { mantenimientos de hardware y software? }\end{array}$ & 100 & Alto \\
\hline \multirow[t]{2}{*}{1.14} & ¿Se realiza evaluación y documentación del impacto de los cambios? & 100 & Alto \\
\hline & Total Promedio & 100 & Alto \\
\hline
\end{tabular}




\begin{tabular}{|c|c|c|c|}
\hline & DOCUMENTACIÓN & Valor & Riesgo \\
\hline 2.1 & $\begin{array}{l}\text { ¿Existe un adecuado archivo de documentos en su área que le permitan } \\
\text { la conservación, consulta y seguridad de los mismos? }\end{array}$ & 32 & Bajo \\
\hline 2.2 & ¿Existen una reglamentación para uso y manejo de la documentación? & 100 & Alto \\
\hline \multirow[t]{2}{*}{2.3} & $\begin{array}{l}\text { ¿Hay procedimientos para corroborar exactitud y compatibilidad de la } \\
\text { documentación? }\end{array}$ & 32 & Bajo \\
\hline & Total Promedio & 54,6666667 & Medio \\
\hline 3. & MANUALES & Valor & Riesgo \\
\hline 3.1 & ¿Usted conoce si existen manuales aplicados a su área en la entidad? & 60 & Medio \\
\hline 3.2 & ¿Dichos manuales están actualizados y acordes con la estructura vigente? & 60 & Medio \\
\hline \multirow[t]{2}{*}{3.3} & $\begin{array}{l}\text { ¿Considera que los manuales de procedimientos y procesos son } \\
\text { herramientas que facilitan la ejecución correcta, oportuna y permanente } \\
\text { de las funciones encomendadas? }\end{array}$ & 60 & Medio \\
\hline & Total Promedio & 60 & Medio \\
\hline 4. & COMUNICACIÓN & Valor & Riesgo \\
\hline 4.1 & $\begin{array}{l}\text { ¿Cree que hay efectividad en la comunicación de los deberes, } \\
\text { obligaciones y responsabilidades respecto a su trabajo? }\end{array}$ & 33 & Bajo \\
\hline 4.2 & $\begin{array}{l}\text { ¿Considera que los deberes y obligaciones de su actividad contribuyen al } \\
\text { logro de as objetivos? }\end{array}$ & 33 & Bajo \\
\hline \multirow[t]{3}{*}{4.3} & $\begin{array}{l}\text { ¿Existe una manera de comunicar los problemas e inquietudes de una } \\
\text { manera inmediata? }\end{array}$ & 33 & Bajo \\
\hline & Total Promedio & 33 & Bajo \\
\hline & TOTAL COMPONENTES INFORMACIÓN Y COMUNICACIÓN & 61,9166667 & Medio \\
\hline
\end{tabular}

Componente 5 - Monitoreo

Los controles que han sido diseñados pueden ser vulnerados, por esta situación se deben monitorear permanentemente que los mecanismos diseñados sigan funcionando adecuadamente y en caso de no ser así, realizar los ajustes respectivos de forma oportuna.

El monitoreo es el componente con el nivel de riesgo mayor, un nivel de riesgo del $100 \%$ indica ausencia total de mecanismos de control asociados a este componente como son la falta de evaluaciones periódicas, la falta de evaluación a la comunicación organizacional, la ausencia de capacitación y monitoreo al trabajo realizado.
Evaluación física al proceso de tesorería

Del resultado de esta encuesta se tomaron 20 empresas a las que se realizó análisis físico a sus procesos en el departamento de Tesorería específicamente en los procesos de: Pago de obligaciones, Recaudo de efectivo, Cuentas por pagar, Cuentas por pagar. Analizando en estas actividades, cuáles son los principales riesgos encontrados, sus causas y consecuencias para las organizaciones, así como la revisión de si el riesgo analizado ya se ha no materializado.

El análisis encontrado es el siguiente: 
- Se evidencia en el proceso de pago de obligaciones de las pequeñas y medianas empresas de Medellín dos riesgos importantes, el fraude y el error en pagos, originados por elementos como el no tener políticas de pagos, la falta de liquidez, la falta de segregación de funciones y la negligencia.

- El procedimiento de recaudo de efectivo también se evidencia el riesgo de fraude en las pequeñas y medianas empresas de Medellín, esto se origina por factores como el incumplimiento en las políticas de seguridad organizacional y por el vacío en procedimientos de control interno, al igual que por la falta de segregación de funciones.

- En el proceso de cuentas por cobrar y por pagar se muestran dos riesgos que prevalecen como el fraude y la pérdida de recursos, originados por falencias en el sistema de control por descontar gastos inexistentes y por falta de gestión, así como falta de otros elementos del control como políticas para el manejo de soportes.

Tabla 7. Componente 5 - Monitoreo organizacional

Table 7. Component 5 - Organizational monitoring

\begin{tabular}{|c|c|c|c|}
\hline 1. & MONITOREO INTERACTIVO & Valor & Riesgo \\
\hline 1.1 & $\begin{array}{l}\text { ¿Se llevan a cabo evaluaciones periódicas y sistemáticas del control } \\
\text { interno? }\end{array}$ & 100 & Alto \\
\hline 1.2 & $\begin{array}{l}\text { ¿Considera que las evaluaciones son efectuadas por personal con } \\
\text { experiencia y conocimientos adecuados? }\end{array}$ & 100 & Alto \\
\hline 1.3 & $\begin{array}{l}\text { ¿Monitorea la compañía en forma rutinaria los controles en el momento } \\
\text { en que se desarrollan las operaciones de la organización? }\end{array}$ & 100 & Alto \\
\hline 1.4 & $\begin{array}{l}\text { ¿Están asignadas las responsabilidades en cuenta a la capacitación y } \\
\text { monitoreo de los controles internos? }\end{array}$ & 100 & Alto \\
\hline 1.5 & $\begin{array}{l}\text { ¿Las fallas detectadas en las evaluaciones son comunicadas y se corrigen } \\
\text { oportunamente? }\end{array}$ & 100 & Alto \\
\hline 1.6 & $\begin{array}{l}\text { ¿Los ambientes internos y externos son monitoreados para obtener } \\
\text { información que pueda señalar una necesidad de reevaluar los objetivos } \\
\text { o controles de la organización? }\end{array}$ & 100 & Alto \\
\hline & Total Promedio & 100 & Alto \\
\hline & TOTAL COMPONENTE MONITOREO & 100 & Alto \\
\hline
\end{tabular}

\section{RESULTADOS Y DISCUSIÓN}

La revisión del control interno en las empresas seleccionadas permitió identificar las debilidades significativas en cada uno de los componentes del control interno. La Figura 1 refleja con claridad dicha situación.

Dentro de la revisión al sistema de control interno de las pequeñas y medianas empresas de Medellín, se encuentran los siguientes resultados:

Los controles internos evaluadas son deficientes, por lo tanto, la gerencia no podrá evaluar con acierto su desempeño frente a las metas que se trace, tanto así que le será difícil proyectarse en crecer y transformarse, ya que no tiene la facilidad de identificar ventajas y desventajas en cada cambio. 
Las organizaciones no cuentan con un buen sistema de control interno implementado en el departamento de Tesorería, observándose fallas en los componentes del COSO, se reitera la importancia que el proceso de control interno identifique $\mathrm{y}$ analice los riesgos relevantes para el logro de los objetivos y la base para determinar la forma en que tales riesgos deben ser manejados, así como el desarrollo de mecanismos de control, métodos de respuesta a las desviaciones encontradas en el sistema y la creación de un mapa o una matriz que permita no solo identificar sino a administrar los riesgos organizacionales.

Figura 1. Valoración del nivel de riesgo

Figure 1. Risk level assessment

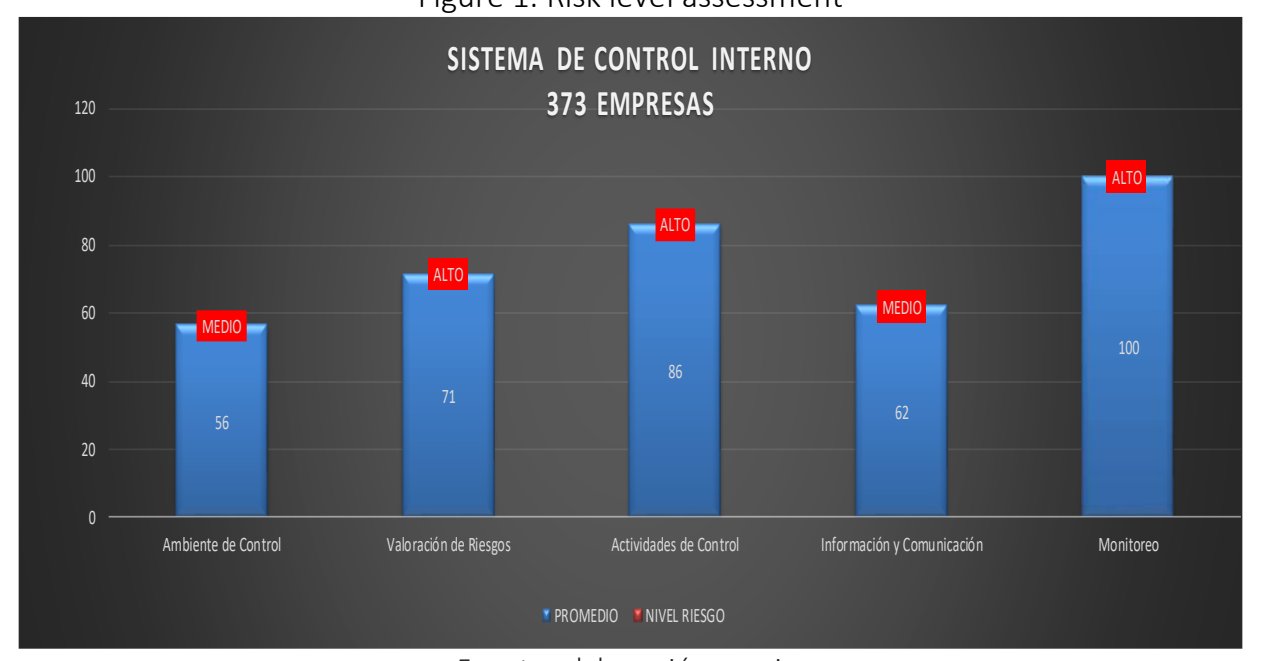

Fuente: elaboración propia.

El manejo que se le está dando al sistema de control interno en el proceso de tesorería en las pequeñas y medianas empresas en la ciudad de Medellín después de aplicar la herramienta de esta investigación demuestra que es débil, es necesario que las empresas le den mayor prioridad a su estructura de control de manera que se permita una adecuada salvaguarda de activos y un mejor nivel de confianza a los dueños de las organizaciones. Al respecto de los vacíos que se presentan en el control interno (PricewaterhouseCoopers, 2016) expresa:

A nivel global se observa una disminución en la efectividad de los esquemas de detección de actividades criminales en términos de control de gestión (detección durante controles corporativos que se ha reducido en un $7 \%$ ). Además, una de cada cinco organizaciones (22\%) a nivel global no ha llevado a cabo ningún tipo de evaluación de los riesgos de fraude en los últimos 24 meses. En Colombia la estadística no está lejos (19\%).

La problemática en el control interno en las organizaciones es evidenciada tanto en la investigación realizada en el municipio de Medellín, como a nivel global, con los resultados arrojados por el trabajo de la Price Watherhouse.

Las pymes no cuentan con un sistema de administración bien definido, así como con su sistema de control interno, en el cual los objetivos y metas en la mayoría de veces no se encuentran escritos, sino que se estructuran solo en la práctica, solucionando problemas a medida que se presentan, evidenciando deficiencias por falta de planificación y estructura organizacional. 


\section{CONCLUSIONES}

Se puede identificar que el componente monitoreo es el que presenta mayor nivel de riesgo, ya que para las empresas evaluadas el $100 \%$ de las respuestas evidencian que no existe monitoreo, esta situación es grave desde la estructura de control interno en el proceso de tesorería, pues la falta de revisiones periódicas, verificación de la forma en que opera el sistema y la necesidad de mejora, generan un nivel de riesgo alto para el control de los recursos organizacionales.

En las pequeñas y medianas empresas, se puede evidenciar que tres de los componentes de control interno evaluados en el proceso de tesorería presentan riesgo alto (valoración de riesgos con un $71 \%$, actividades de control con un $86 \%$ y monitoreo con un $100 \%$ ) originando debilidades importantes, que necesitan una urgente atención, esta situación denota la probabilidad alta de pérdida de recursos organizacionales y la necesidad de generar mecanismos de control que garanticen la salvaguarda de los activos de los inversionistas.

Es necesario sensibilizar a la alta gerencia sobre la importancia del control interno en las organizaciones, así mismo en compañía con el jefe de control interno o quien haga sus veces incrementar la cultura hacia el control en sus colaboradores, se identifica que el concepto de control interno para el proceso de tesorería en la mayoría de los casos se presume que no necesita mejoras, incrementando con esto riesgos como el fraude, el robo y en general la perdida de activos, imagen y confianza por parte posibles inversionistas en la organización, lo que hace necesario que se realice un control constante en esta dependencia para prevenir la materialización de los riesgos inherentes a la operación.

\section{REFERENCIAS}

Argente, E. (2012). El gobierno corporativo en empresas hispano marroquíes. Granada: Universidad de Granada. Recuperado de: https://hera.ugr.es/tesisugr/20948670.p df

Cámara de Comercio de Medellín para Antioquia (2016). Estadísticas Cámara. Recuperado de http://www.camaramedellin.com.co/site /Servicios-Empresariales/InformacionEmpresarial/Estadisticas-Camara.aspx

Capote, G. (2000). El control interno y el control. Economía y Desarrollo, 129(2).

Cartategui, M. (2009). Debilidades en el ambiente del trabajo que afectan el control interno. Argentina: El Cid.

COSO (2016). Enterprise Risk Management. Aligning Risk with Strategy and Performance. Recuperado de https://www.coso.org/Documents/COSO -ERM-draft-Post-Exposure-Version.pdf

Departamento Administrativo de la Función Pública. (2014). Manual Técnico del Modelo Estándar de Control Interno para el Estado Colombiano MECI 2014. Recuperado de https://www.procuraduria.gov.co/portal/ media/file/MECl_2014.pdf

Estupiñán, R. (2015). Control interno y fraudes: análisis de informe COSO I, II y III con base en los ciclos transaccionales. Bogotá: Ecoe Ediciones.

Lara, M. I. (2007). Manual básico de revisión y verificación contable. Madrid: DYKINSON. 
Ortiz, G., Esquivel, E., \& Hernández, O. (2016). El impacto de la relación con el cliente y de la capacidad de valor agregado en el servicio en el rendimiento de la pyme manufacturera de Aguascalientes. Revista CEA, 2(4), 47-58.
Vélez, F. M., \& Duque, G. A. (2017). Gestión de una comunidad de práctica. Caso de estudio: incidentes de infraestructura de dos pymes de la ciudad de Medellín. Revista CEA, 3(5), 59-68. 
